\section{Bioelectrical Impedance Analysis Overestimates Fat-Free Mass in Breast Cancer Patients Undergoing Treatment}

\author{
Kirsten Elizabeth Bell, PhD ${ }^{1}$ (D); Schuyler Schmidt, BSc ${ }^{1}$; Amanda Pfeiffer, MSc ${ }^{1}$; \\ Lisa Bos, BASc, RD ${ }^{1}$; Carrie Earthman, PhD, RD, LD ${ }^{2}$; Caryl Russell, MSc ${ }^{1}$; \\ and Marina Mourtzakis, $\mathbf{P h D}^{1}$
}

Volume 00 Number 0

xxxx $20191-12$

(C) 2019 American Society for

Parenteral and Enteral Nutrition

DOI: $10.1002 /$ ncp. 10438

wileyonlinelibrary.com

WILEY

\begin{abstract}
Background: Bioelectrical impedance analysis (BIA) is commonly used to assess fat-free mass (FFM) and fat mass (FM) in breast cancer patients. However, because of the prevalence of overweight, obesity and variable hydration status in these patients, assumptions for existing prediction equations developed in healthy adults may be violated, resulting in inaccurate body composition assessment. Methods: We measured whole-body FFM using single-frequency BIA $(50 \mathrm{kHz})$ and dual-energy x-ray absorptiometry (DXA) in 48 patients undergoing treatment for breast cancer. We applied raw BIA data to 18 previously published FFM prediction equations (FFM $\left.\mathrm{BIA}_{\mathrm{A}}\right)$ and compared these estimates to DXA (FFM $\mathrm{DXA}_{\text {; }}$; reference method). Results: On average, patients were $52 \pm$ 10 (mean \pm SD) years of age and overweight (body mass index: $27.5 \pm 5.5 \mathrm{~kg} / \mathrm{m}^{2}$; body fat by DXA: $40.1 \% \pm 6.6 \%$ ). Relative to DXA, BIA overestimated FFM by $4.1 \pm 3.4 \mathrm{~kg}\left(\mathrm{FFM}_{\mathrm{DXA}}: 42.0 \pm 5.9 \mathrm{~kg}\right.$; FFM $\left._{\mathrm{BIA}}: 46.1 \pm 3.4 \mathrm{~kg}\right)$. Individual equationgenerated predictions of $\mathrm{FFM}_{\mathrm{BIA}}$ ranged from $39.6 \pm 6.7$ to $52.2 \pm 5.6 \mathrm{~kg}$, with 16 equations overestimating and 2 equations underestimating $\mathrm{FFM}_{\mathrm{BIA}}$ compared with $\mathrm{FFM}_{\mathrm{DXA}}$. Based on equivalence testing, no equation-generated estimates were equivalent to DXA. Conclusion: Compared with DXA, BIA overestimated FFM in breast cancer patients during treatment. Although several equations performed better than others, none produced values that aligned closely with DXA. Caution should be used when interpreting BIA measurements in this clinical population, and future studies should develop prediction equations specific to breast cancer patients. (Nutr Clin Pract. 2019;00:1-12)
\end{abstract}

\title{
Keywords
}

adiposity; bioelectrical impedance analysis; body composition; breast neoplasms; dual-energy x-ray absorptiometry; skeletal muscle

\section{Introduction}

Approximately two-thirds of recently diagnosed breast cancer patients have overweight or obesity. ${ }^{1,2}$ Further, patients can gain up to $5 \mathrm{~kg}$ body mass during treatment $\mathrm{t}^{3,4}$ and may continue to gain weight for several years into survivorship. ${ }^{5}$ Excess adiposity is associated with greater breast cancerspecific and all-cause mortality, ${ }^{6}$ impaired effectiveness of certain forms of treatment, ${ }^{7-9}$ and increased risk of developing secondary morbidities (such as cardiovascular disease, diabetes, and cancer recurrence) in survivorship. Even patients who do not gain weight during treatment may exhibit deleterious body composition changes (ie, concomitant losses of fat-free mass [FFM] and gains in fat mass [FM] with no net change in body mass) that contribute to adverse disease-specific and overall health outcomes. ${ }^{10}$ Weight management through exercise and nutrition programs has become an important component of breast cancer rehabilitation, ${ }^{6,11}$ and practical evaluation of changes in body composition features as they relate to the disease trajectory, or as a measure of efficacy of nutrition and exercise programs, is needed in this patient population.
Bioelectrical impedance analysis (BIA) is the most widely used body composition modality in clinical settings ${ }^{12}$ because of its accessibility, ease of use, and noninvasive nature. BIA functions by passing a small electric current at 1 (single-frequency BIA [SF-BIA], usually at $50 \mathrm{kHz}$ ) or more (multifrequency BIA [MF-BIA] or bioimpedance

From the ${ }^{1}$ Department of Kinesiology, University of Waterloo, Waterloo, Ontario, Canada; and the ${ }^{2}$ Department of Behavioral Health and Nutrition, College of Health Sciences, University of Delaware, Newark, Delaware, USA.

Financial disclosure: This work was funded by a Canadian Institutes of Health Research grant, an Ontario Ministry of Research and Innovation Early Researcher Award, and the Canadian Foundation for Innovation (all to M. Mourtzakis).

Conflicts of interest: None declared.

This article originally appeared online on xxxx 0, 2019.

Corresponding Author:

Marina Mourtzakis, PhD, Department of Kinesiology - BMH 3033 , University of Waterloo, 200 University Avenue West, Waterloo, ON N2L 3G1, Canada.

Email:mmourtza@uwaterloo.ca 
spectroscopy [BIS]) frequencies through the body and monitoring the reduction in voltage. The impedance characteristics of the current ( $R$, resistance; $X$, reactance) are then used to estimate total body water (TBW), from which FFM (water-based tissues through which the electrical current is easily conducted) and FM (tissues that impede the current), or percent body fat, can be calculated. However, SF-BIA and MF-BIA rely entirely on prediction equations to estimate TBW (and FFM) from $R$ and $X$, and the validity and accuracy of each equation is highly dependent on the characteristics of the population in which it was developed (eg, age, sex, ethnicity). BIS, in contrast, is based on biophysical modeling. Because BIS does not require the use of regression-derived prediction equations, it is less vulnerable to the population-specific assumptions of SFBIA and MF-BIA, ${ }^{12}$ although there are several constants in commonly applied BIS algorithms that can be sources of error. Furthermore, all forms of BIA (including BIS) are negatively influenced by abnormal body geometry (as is often observed in individuals with overweight and obesity) and atypical fluid distribution of the patient. ${ }^{13}$

BIA is commonly used to evaluate body composition in breast cancer patients; but despite that, no breast cancerspecific prediction equations exist. Because breast cancer patients often have overweight or obesity and sometimes develop lymphedema (swelling and fluid retention) as a result of treatment, they possess unique body geometry and fluid shifts that may violate the assumptions of available equations derived from healthy, normal-weight populations. Therefore, the use of BIA to assess body composition in breast cancer patients may produce inaccurate estimations.

The objective of this study was to compare the ability of previously published SF-BIA equations that predict FFM with measurements of FFM made using a reference method (dual-energy x-ray absorptiometry [DXA]) in a group of breast cancer patients currently undergoing treatment. We hypothesized that most BIA equations would overestimate FFM and be unsuitable for use in these patients.

\section{Methods}

\section{Subjects}

Forty-eight female breast cancer patients took part in the present study. These patients were part of a larger trial that evaluated the effect of an exercise training program on body composition and metabolic outcomes (M. Mourtzakis, unpublished data). Any female currently undergoing treatment for breast cancer was eligible to participate, regardless of disease stage, treatment stage, or treatment type. This study received clearance from the university's ethics board (ORE \#: 18987). All participants were informed of the nature and possible risks associated with the study before their written informed consent was obtained.

\section{Study Protocol}

During a single study visit, participants reported to the laboratory after 8-12 hours of overnight fasting and received a whole-body DXA scan followed by 2 wrist-ankle SF-BIA measurements.

\section{Dual-Energy X-Ray Absorptiometry}

Whole-body FFM, FM, fat- and bone-free mass (FBFM; ie, lean soft tissue), and bone mineral content were measured using DXA (Hologic Discovery QDR 4500, Christie Group, Mississauga, ON, Canada). Prior to the DXA scan, participants changed into a cloth hospital gown, and weight and height were obtained using a balance-beam scale and stadiometer, respectively. Participants were then positioned supine on the scanning table with their shoulders depressed and forearms positioned parallel to the bed. Participants' legs were extended with their toes internally rotated and held in position with masking tape to prevent movement during the scan. A second scan was required for participants $(n=2)$ who did not fit within the lateral limits of the scanning table. These scans were analyzed by summing the left and right segments of the body bisected along the midline of the spine, as previously described. ${ }^{14}$ All scans were performed and analyzed by 1 of 2 certified medical radiation technologists (MRTs). Prior to all scans, the MRTs performed quality control and phantom calibration procedures using whole-body and spine phantoms.

\section{Bioelectrical Impedance Analysis}

Raw $R$ and $X$ measurements at $50 \mathrm{kHz}$ were obtained using the BIA Quantum IV (RJL Systems, Clinton TWP, MI, USA). After voiding their bladder and removing all jewelry, participants lay supine while the electrode sites were cleaned with rubbing alcohol or iodine (if a patient was sensitive to alcohol). Four electrodes were then placed on the right side of the body in the following locations: (1) hand (dorsal side of the third metacarpal-phalangeal joint), (2) wrist (on the dorsal side between the styloid processes of the radius and ulna), (3) foot (dorsal side of the second and third metatarsal-phalangeal joints), and (4) ankle (on the anterior side between the lateral and medial malleoli). Arms were abducted $30^{\circ}$ from the trunk, and the feet were not touching. Participants lay supine for a minimum of 5 minutes to allow for fluid shifts prior to obtaining measurements. Measurements were performed twice, and the average $R$ and $X$ values were used to calculate FFM $_{\text {BIA }}$.

\section{Waist Circumference}

A tape measure was used to measure waist circumference at the top of the iliac crests following a normal expiration, while participants stood with their arms crossed over the chest and feet shoulder-width apart. 


\section{Selection of Prediction Equations}

We searched PubMed and Google Scholar for equations to predict whole-body FFM or TBW from SF-BIA measurements $(50 \mathrm{kHz})$ that were developed and validated against a reference method (isotope dilution, hydrodensitometry, multiple-component models, or DXA). To minimize error, we only included equations that used wrist-ankle SF-BIA devices. In an effort to match our patient sample as closely as possible, we also excluded equations that were developed and/or validated in children and adolescents, male-only populations, exclusively non-Caucasian populations, clinical populations, and populations from developing countries.

\section{Estimation of FFM by BIA}

To generate estimates of FFM $_{\text {BIA }}$, we applied the raw $R$ and $X$ values from BIA measurements made in our sample of breast cancer patients to the selected prediction equations. For equations that required impedance $(Z), Z$ was calculated as follows:

$z=\sqrt{\left(R^{2}+X^{2}\right)}$

\section{Statistical Analysis}

Several statistical tests may be used to evaluate the agreement between 2 methods; however, there are limitations inherent to each approach. Based on recent recommendations aimed at addressing this criticism, ${ }^{12}$ we compared estimates of $\mathrm{FFM}_{\mathrm{BIA}}$ to $\mathrm{FFM}_{\mathrm{DXA}}$ using multiple statistical techniques. First, we used 2-tailed paired Student $t$-tests to assess whether estimates of FFM BIA were significantly different than $\mathrm{FFM}_{\mathrm{DXA}}$ measurements. We tested for equivalence between the 2 methods using the $90 \%$ confidence interval (CI) method. ${ }^{15}$ Coefficients of variation (CVs) for FFM measurements made in women with overweight and obesity using DXA range from $0.94 \%$ to $1.34 \% .{ }^{16-18}$ Therefore, we used the median reported $\mathrm{CV}$ of $1.30 \%$ to establish an equivalence range of -0.504 to 0.504 $\mathrm{kg}$. This range was used when conducting the equivalence tests. Next, we used Lin's concordance correlations $\left(r_{\mathrm{c}}\right)$ to evaluate the strength of the relationship between FFM $_{\text {BIA }}$ and FFM $\mathrm{DXA}_{\mathrm{DA}}$. Lastly, we constructed Bland-Altman plots to test for systematic error associated with BIA. A mean difference between the 2 methods (ie, fixed bias) of $<0$ or $>0$ would suggest that BIA consistently underestimates or overestimates FFM, respectively. ${ }^{19}$ We also tested for evidence of proportional bias by performing Pearson productmoment correlations between the mean of the 2 methods

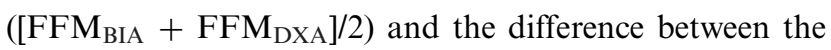
2 methods $\left(\mathrm{FFM}_{\mathrm{BIA}}-\mathrm{FFM}_{\mathrm{DXA}}\right)$. A significant correlation between these variables indicates proportional bias.

In an exploratory secondary analysis, we divided our cohort of 48 patients into the following body mass index
(BMI) categories: normal $\left(18.5-24.9 \mathrm{~kg} / \mathrm{m}^{2}, \mathrm{n}=18\right)$, overweight $\left(25-29.9 \mathrm{~kg} / \mathrm{m}^{2}, \mathrm{n}=16\right)$, obese $\left(30-35 \mathrm{~kg} / \mathrm{m}^{2}\right.$, $\mathrm{n}=11)$, and severely obese $\left(>35.0 \mathrm{~kg} / \mathrm{m}^{2}, \mathrm{n}=3\right)$. Using a 1-way analysis of variance, we examined whether FFM $_{\text {DXA }}, F_{F} M_{\text {BIA }}$, and the difference between the 2 methods $\left(\mathrm{FFM}_{\mathrm{BIA}}-\mathrm{FFM}_{\mathrm{DXA}}\right)$ were different among these 4 subgroups.

Statistical analysis was conducted using IBM SPSS Statistics version 25.0 for Mac (Armonk, NY, USA) and GraphPad Prism 8.0 (La Jolla, CA, USA). Significance was accepted as $P \leq .05$. Data are presented as mean $\pm \mathrm{SD}$.

\section{Ranking of Equations}

To objectively select equations that produced accurate estimates of $\mathrm{FFM}_{\mathrm{BIA}}$ in this patient population, we assigned a point when each of the following 6 criteria were met: (1) $P>.05$ during the paired $t$-test, (2) strong correlation $\left(r_{\mathrm{c}}>0.80\right)$, (3) equivalence (ie, $90 \%$ CI for the mean difference contained within the equivalence region of -0.504 to $0.504 \mathrm{~kg}$ ), (4) fixed bias within the CV between repeat DXA measurements $(0.0 \pm 0.504 \mathrm{~kg})$, (5) limits of agreement (ie, $1.96^{*} \mathrm{SD}$ of the mean difference between the methods) within $\pm 10 \%$ of the group mean FFM $_{\mathrm{DXA}}$ (42 $\mathrm{kg}$ ), and (6) no proportional bias during Bland-Altman analysis. There is no consensus on the acceptable range for the limits of agreement, ${ }^{12}$ but previous studies have considered $\pm 5 \%$ to $10 \%$ as wide. ${ }^{20}$ As such, we have used $\pm 10 \%$ as our acceptable range for limits of agreement. We then tallied the points to determine which equations were most, and least, likely to provide valid estimates of FFM using BIA in this group of breast cancer patients.

\section{Results}

\section{Patients}

On average, patients were $52 \pm 10$ years old (range: 30 75 years) and had $40.1 \% \pm 6.6 \%$ body fat (range: $23.8 \%-$ $49.3 \%$; Table 1) as measured by DXA. Most patients had overweight or obesity, based on BMI $(n=30$, or $63 \%)$, and central obesity according to waist circumference $(n=29$, or $62 \%$ ), which is in line with data on larger breast cancer populations. ${ }^{1,2}$

Most patients ( $n=44$, or $92 \%$ ) presented with unilateral breast cancer, and the side affected was split approximately evenly between right and left. Surgery (mastectomy, lumpectomy, or wedge resection; $88 \%$ of patients, Table 2) and radiation therapy ( $83 \%$ of patients) were the most common forms of treatment. Axillary or sentinel lymph node dissection was performed in $22(46 \%)$ patients. Seventy-one percent of patients received chemotherapy, and the majority $(63 \%)$ of chemotherapy was taxane-based. Hormonal therapy and targeted (ie, monoclonal antibody) treatment was provided to $63 \%$ and $21 \%$ of patients, respectively. 
Table 1. Physical and Clinical Characteristics of Patients $(\mathrm{n}=$ 48).

\begin{tabular}{|c|c|}
\hline Characteristics & Mean \pm SD (Range) \\
\hline Age, y & $52 \pm 10(30-75)$ \\
\hline Weight, $\mathrm{kg}$ & $72.7 \pm 14.1(49.1-102.0)$ \\
\hline Height, m & $1.63 \pm 0.07(1.51-1.80)$ \\
\hline BMI, $\mathrm{kg} / \mathrm{m}^{2}$ & $27.5 \pm 5.5(18.9-41.8)$ \\
\hline Underweight, $<18.5 \mathrm{~kg} / \mathrm{m}^{2}(\mathrm{n}, \%)$ & $0,0 \%$ \\
\hline Normal, $18.5-24.9 \mathrm{~kg} / \mathrm{m}^{2}$ (n, $\left.\%\right)$ & $18,38 \%$ \\
\hline Overweight, $25-29.9 \mathrm{~kg} / \mathrm{m}^{2}(\mathrm{n}, \%)$ & $16,33 \%$ \\
\hline Obese, $30-35 \mathrm{~kg} / \mathrm{m}^{2}(\mathrm{n}, \%)$ & $11,23 \%$ \\
\hline Severely obese, $>35 \mathrm{~kg} / \mathrm{m}^{2}(\mathrm{n}, \%)$ & $3,6 \%$ \\
\hline Waist circumference, $\mathrm{cm}^{\mathrm{a}}$ & $94.1 \pm 13.5(71-122)$ \\
\hline$>88 \mathrm{~cm}(\mathrm{n}, \%)$ & $29,62 \%$ \\
\hline $\mathrm{FM}_{\mathrm{DXA}}(\%)$ & $40.1 \pm 6.6(23.8-49.3)$ \\
\hline $\mathrm{FM}_{\mathrm{DXA}}, \mathrm{kg}$ & $29.1 \pm 9.3(11.6-47.5)$ \\
\hline $\mathrm{FFM}_{\mathrm{DXA}}, \mathrm{kg}$ & $42.0 \pm 5.9(32.1-55.6)$ \\
\hline $\mathrm{FBFM}_{\mathrm{DXA}}, \mathrm{kg}$ & $39.8 \pm 5.7(30.7-52.7)$ \\
\hline $\mathrm{BMC}_{\mathrm{DXA}}, \mathrm{kg}$ & $2.2 \pm 0.4(1.4-3.0)$ \\
\hline \multicolumn{2}{|l|}{ Side affected $(n, \%)$} \\
\hline Right & $19,40 \%$ \\
\hline Left & $25,52 \%$ \\
\hline Bilateral & $2,4 \%$ \\
\hline \multicolumn{2}{|l|}{ Evidence of lymphedema (n, \%) } \\
\hline Yes & $2,4 \%$ \\
\hline No & $46,96 \%$ \\
\hline \multicolumn{2}{|l|}{ Ethnicity (n, \%) } \\
\hline Caucasian & $47,98 \%$ \\
\hline Asian & $1,2 \%$ \\
\hline
\end{tabular}

BMI, body mass index; BMC, bone mineral content; DXA, dualenergy x-ray absorptiometry; FBFM, fat- and bone-free mass; FFM, fat-free mass; FM, fat mass.

${ }^{\mathrm{a}} \mathrm{n}=47$.

Evidence of lymphedema was reported in 2 patients. Please refer to Supplementary Table S1 for additional medications prescribed to patients.

\section{Prediction Equations}

We identified 18 prediction equations from 13 source articles, which are presented in Table 3. The majority of equations (14/18 equations) were developed using SF-BIA instruments from RJL Systems. Of the remaining equations, 2 were developed using the Quadscan 4000 (Bodystat; Isle of Man, UK), 1 used the Bodystat 1500 (Bodystat; Isle of Man, UK), and 1 used the Human-IM Plus (Dietosystem; Milan, Italy). Hydrodensitometry was the most commonly employed reference technique (7/18 equations); other reference techniques included DXA (5 equations), multiplecomponent models (4 equations), and deuterium dilution ( 2 equations).

The populations in which the equations were developed and validated were either American (6 sources; see Table 4) or European ( 7 sources) and entirely Caucasian, except for 1 study whose subject pool was $20 \%$ African American. ${ }^{21}$ The majority of studies evaluated healthy adult popula-
Table 2. Type of Cancer Treatment Administered to Patients $(\mathrm{n}=48)$.

\begin{tabular}{lc}
\hline Treatment Type & $\mathrm{n}(\%)$ \\
\hline Surgery & $42(88)$ \\
Lumpectomy & $21(44)$ \\
Mastectomy & $15(31)$ \\
Wedge resection & $5(10)$ \\
Unspecified & $1(2)$ \\
Lymph node dissection & $22(46)$ \\
Radiation & $40(83)$ \\
Chemotherapy & $34(71)$ \\
ACT & $21(44)$ \\
CT & $4(8)$ \\
AC & $1(2)$ \\
Taxol only & $3(6)$ \\
Docetaxel and carboplatin & $2(4)$ \\
FEC-D & $1(2)$ \\
Unspecified & $2(4)$ \\
Hormonal therapy & $30(63)$ \\
Tamoxifen & $12(25)$ \\
Anastrozole & $6(13)$ \\
Letrozole & $5(10)$ \\
Exemestane & $1(2)$ \\
Unspecified & $8(17)$ \\
Targeted therapy & $10(21)$ \\
Trastuzumab & $10(21)$ \\
Pertuzumab & $2(4)$ \\
\hline
\end{tabular}

$\mathrm{AC}$, doxorubicin and cyclophosphamide; ACT, doxorubicin (Adriamycin) and cyclophosphamide (Cytoxan), followed by paclitaxel (Taxol) or docetaxel (Taxotere); CT, cyclophosphamide and docetaxel; FEC-D, 5-fluorouracil, epirubicin, and cyclophosphamide, followed by docetaxel.

tions (11/13 sources) comprising both sexes ( $9 / 13$ sources); 2 sources only included obese adults, and 4 sources only included females.

\section{Estimation of FFM by BIA}

On average, the selected equations predicted FFM $_{\text {BIA }}$ to be $46.1 \pm 3.4 \mathrm{~kg}$ in our sample of breast cancer patients. Relative to $\mathrm{FFM}_{\text {DXA }}(42.0 \pm 5.9 \mathrm{~kg})$, the equations overestimated $\mathrm{FFM}_{\text {BIA }}$ by $4.1 \pm 3.4 \mathrm{~kg}$ (Table 5). Individual equation-generated predictions of $\mathrm{FFM}_{\mathrm{BIA}}$ ranged from $39.6 \pm 6.7$ to $52.2 \pm 5.6 \mathrm{~kg}$, with 16 equations overestimating and 2 equations underestimating FFM $_{\text {BIA }}$ compared with $F_{\text {FFMA }}$. The technical error measurement associated with our BIA instrument is $2.14 \Omega(0.42 \%)$ for $R$ and $1.94 \Omega$ $(3.52 \%)$ for $X$.

\section{Paired $t$-Tests and Equivalence Tests}

Except for equations 6 and 10, all equations produced estimates of FFM $_{\text {BIA }}$ that were significantly different from FFM $_{\text {DXA }}$ (Table 5). Using the $90 \%$ CI method, no equations produced estimates of FFM were equivalent to DXA measurements (Figure 1). 
Table 3. BIA Prediction Equations Evaluated in This Study.

\begin{tabular}{|c|c|c|c|c|}
\hline \multicolumn{2}{|c|}{$\begin{array}{l}\text { Source (First } \\
\text { Author, Date) }\end{array}$} & Equation & \multirow{2}{*}{$\begin{array}{c}\text { Instrument } \\
\text { Human-IM Plus }\end{array}$} & \multirow{2}{*}{$\begin{array}{l}\text { Reference Technique } \\
\text { DXA (Lunar Prodigy) }\end{array}$} \\
\hline 1 & $\begin{array}{l}\text { Bedogni } \\
2015\end{array}$ & $\begin{array}{l}\mathrm{FFM}=(-7 \times \mathrm{PWS})+\left(0.57 \times\left(\mathrm{ht}^{2} / \mathrm{Z}\right)\right)+22.07 \\
(\text { no }=0 ; \text { yes }=1)\end{array}$ & & \\
\hline 2 & Ramel 2011 & $\begin{array}{l}F F M=7.610-(0.0855 \times \text { age })+(0.273 \times w t)+ \\
\quad(0.148 \times h t)-(0.00746 \times R)\end{array}$ & Bodystat 1500 & $\begin{array}{l}\text { DXA (Hologic QDR } \\
2000 \text { plus) }\end{array}$ \\
\hline 3 & $\begin{array}{l}\text { Verdich } \\
2011^{\mathrm{a}}\end{array}$ & $\begin{array}{l}\text { a) } F F M=\left(0.314 \times\left(\mathrm{ht}^{2} / \mathrm{Z}_{50}\right)\right)+(0.174 \times \mathrm{wt})+ \\
(0.143 \times \text { age })+12.1 \\
\text { b) } \mathrm{FFM}=\left(0.813 \times\left(\mathrm{ht}^{2} / \mathrm{Z}_{50}\right)\right)+8.91\end{array}$ & Quadscan $4000^{\mathrm{f}}$ & $\begin{array}{l}\text { DXA (Lunar DPX-IQ } \\
\text { and Hologic QDR } \\
\text { 2000) }\end{array}$ \\
\hline 4 & Sun 2003 & $F F M=-9.53+\left(0.69 \times\left(h^{2} / R\right)\right)+(0.17 \times w t)+(0.02 \times R)$ & RJL BIA-101 & Multicomponent model ${ }^{\mathrm{d}}$ \\
\hline 5 & Dey 2003 & $\begin{array}{l}\mathrm{FFM}=11.78+\left(0.499 \times\left(\mathrm{ht}^{2} / \mathrm{R}\right)\right)+(0.134 \times \mathrm{wt})+ \\
\quad(3.449 \times \text { sex }) \\
(\text { female }=0, \text { male }=1)\end{array}$ & RJL BIA-101 & 4 compartment model $^{\mathrm{e}}$ \\
\hline 6 & $\begin{array}{l}\text { Roubenoff } \\
1997\end{array}$ & $\begin{array}{l}\mathrm{FFM}=5.7410+\left(0.4551 \times\left(\mathrm{ht}^{2} / \mathrm{R}\right)\right)+(0.1405 \times \mathrm{wt})+ \\
\quad(0.0573 \times \mathrm{X})+(6.2467 \times \operatorname{sex}) \\
(\text { female }=0 ; \text { male }=1)\end{array}$ & RJL BIA-101 & DXA (Lunar DPX-L) \\
\hline 7 & $\begin{array}{l}\text { Deurenberg } \\
1991\end{array}$ & $\begin{array}{l}\mathrm{FFM}=\left(0.340 \times 10^{4} \times\left(\mathrm{ht}^{2} / \mathrm{R}\right)\right)+(15.34 \times \mathrm{ht})+ \\
\quad(0.273 \times \mathrm{wt})-(0.127 \times \text { age })+(4.56 \times \mathrm{sex})-12.44 \\
(\text { female }=0 ; \text { male }=1)\end{array}$ & RJL BIA-101 & Hydrodensitometry \\
\hline 8 & $\begin{array}{l}\text { Deurenberg } \\
1990\end{array}$ & $\begin{array}{l}\mathrm{FFM}=3.9+\left(0.671 \times 10^{4} \times\left(\mathrm{ht}^{2} / \mathrm{R}\right)\right)+(3.1 \times \mathrm{sex}) \\
(\text { female }=0 ; \text { male }=1)\end{array}$ & RJL BIA-101 & Hydrodensitometry \\
\hline 9 & $\begin{array}{l}\text { Heitmann } \\
1990\end{array}$ & $\begin{array}{l}\text { a) } \mathrm{TBW}=(0.172 \times \mathrm{wt})+\left(0.24 \times\left(\mathrm{ht}^{2} / \mathrm{R}\right)\right)+(0.165 \times \mathrm{ht})- \\
17.58 \\
\text { b) } \mathrm{FFM}=(0.181 \times \mathrm{wt})+\left(0.279 \times\left(\mathrm{ht}^{2} / \mathrm{R}\right)\right)-(0.077 \times \mathrm{age}) \\
+(0.231 \times \mathrm{ht})-14.94\end{array}$ & RJL BIA-103 & 4-compartment model ${ }^{\mathrm{e}}$ \\
\hline 10 & $\begin{array}{c}\text { Lukaski } \\
1988\end{array}$ & $\begin{array}{l}\mathrm{TBW}=\left(0.377 \times\left(\mathrm{ht}^{2} / \mathrm{R}\right)\right)+(0.14 \times \mathrm{wt})-(0.08 \times \text { age })+4.65 \\
\quad+(2.9 \times \text { sex }) \\
(\text { female }=0 ; \text { male }=1)\end{array}$ & RJL BIA-101 & Deuterium dilution \\
\hline 11 & Segal $1988^{b}$ & $\begin{array}{l}\text { a) } F F M=\left(0.00108 \times \mathrm{ht}^{2}\right)-(0.0209 \times \mathrm{R})+(0.23199 \times \mathrm{wt})- \\
(0.06777 \times \text { age })+14.59453 \\
\text { b) } \mathrm{FFM}=\left(0.00091186 \times \mathrm{ht}^{2}\right)-(0.01466 \times \mathrm{R})+ \\
(0.29990 \times \mathrm{wt})-(0.07012 \times \text { age })+9.37938\end{array}$ & $\begin{array}{l}\text { RJL (model not } \\
\text { specified) }\end{array}$ & Hydrodensitometry \\
\hline 12 & $\begin{array}{l}\text { Van Loan } \\
1987\end{array}$ & $\begin{array}{l}\text { a) } \mathrm{TBW}=\left(0.000724 \times \mathrm{ht}^{2}\right)+(0.2822 \times \mathrm{wt})- \\
(0.0153 \times \mathrm{R})-(2.3313 \times \mathrm{sex})-(0.1319 \times \text { age })+9.9868 \\
\text { b) } \mathrm{FFM}=17.7868+\left(0.00085 \times \mathrm{ht}^{2}\right)+(0.3736 \times \mathrm{wt})- \\
(0.02375 \times \mathrm{R})-(0.1531 \times \text { age })-(4.2921 \times \text { sex }) \\
(\text { female }=1 ; \text { male }=0)\end{array}$ & RJL BIA-101 & $\begin{array}{l}\text { Deuterium dilution } \\
\text { (TBW) and } \\
\text { hydrodensitometry } \\
\text { (FFM) }\end{array}$ \\
\hline 13 & $\begin{array}{l}\text { Lukaski } \\
1986^{c}\end{array}$ & $\begin{array}{l}\text { a) } F F M=\left(0.756 \times\left(\mathrm{ht}^{2} / \mathrm{R}\right)\right)+(0.110 \times \mathrm{wt})+ \\
(0.107 \times \mathrm{X})-5.463 \\
\text { b) } \mathrm{FFM}=\left(0.821 \times\left(\mathrm{ht}^{2} / \mathrm{R}\right)\right)+4.917\end{array}$ & RJL BIA-101 & Hydrodensitometry \\
\hline
\end{tabular}

BIA, bioelectrical impedance analysis; DXA, dual-energy x-ray absorptiometry; FFM, fat-free mass in kg; ht, height in cm; PWS, Prader-Willi Syndrome; R, resistance; TBW, total body water in $\mathrm{kg}$; wc, waist circumference in $\mathrm{cm}$; wt, weight in $\mathrm{kg}$; X, reactance; Z, impedance.

${ }^{a}$ Equations (a) and (b) were developed from subjects scanned on a Lunar $(\mathrm{n}=47)$ and Hologic $(\mathrm{n}=84)$ DXA, respectively.

${ }^{b}$ Equation (a) was developed using all subjects; equation (b) was derived from subjects with $>30 \%$ body fat $(\mathrm{n}=175)$, as measured using hydrodensitometry.

${ }^{c}$ Equation (a) was developed using all subjects; equation (b) was developed using only female subjects $(\mathrm{n}=67)$.

${ }^{\mathrm{d}}$ Based on the measurement of body density by hydrodensitometry, TBW by deuterium dilution, and bone mineral content by DXA (Sun et al ${ }^{21}$ used both Lunar [model not specified] and Hologic QDR 2000 scanners), as described by Withers et al. ${ }^{45}$

${ }^{\mathrm{e}}$ Based on the measurement of body weight, TBW by tritium dilution, and body cell mass by $42 \mathrm{~K}$ dilution, as described by Bruce et al. ${ }^{46}$ Note: FFM can be estimated from TBW by assuming hydration constant of (ie, dividing by) 0.732 .

${ }^{\mathrm{f}}$ The Quadscan 4000 is a MF-BIA device, but equations $3 \mathrm{a}$ and $3 \mathrm{~b}$ are specific to the $50-\mathrm{kHz}$ frequency.

\section{Lin's Concordance Correlations}

Based on concordance correlation coefficient ratings by Altman, ${ }^{22}$ we observed an excellent $\left(r_{\mathrm{c}}>0.80\right)$ positive correlation between FFM $_{\text {DXA }}$ and estimates of FFM $_{\text {BIA }}$ made using equation 7 (Figure 2 and Supplementary Figure S2). Estimates from the remaining equations produced weak to moderate $\left(0.20 \leq r_{\mathrm{c}} \leq 0.80\right.$; equations $2,3 \mathrm{a}, 4,5,6,8$, $9 \mathrm{a}$, $9 \mathrm{~b}, 10,11 \mathrm{a}, 11 \mathrm{~b}, 12 \mathrm{a}, 12 \mathrm{~b}, 13 \mathrm{a}$, and 13b) or poor $\left(r_{\mathrm{c}}<0.20\right.$; equations 1 and $3 b$ ) positive correlations with $\mathrm{FFM}_{\mathrm{DXA}}$. 
Table 4. Descriptions of Populations in Which the Predictive Equations Were Developed and Validated.

\begin{tabular}{|c|c|c|c|c|}
\hline & Population & Age, $y$ & $\begin{array}{l}\text { Sex and Sample } \\
\text { Size }\end{array}$ & BMI and Percent Body Fat ${ }^{1}$ \\
\hline 1 & $\begin{array}{l}\text { Obese Italian adult } \mathrm{F} \text { (with } \\
\text { and without PWS) }\end{array}$ & $\begin{array}{l}\text { PWS: } 30 \text { (95\% CI, 27-33) } \\
\text { Without PWS: } 31(95 \% \text { CI, } \\
\quad 28-33)\end{array}$ & $\begin{array}{l}\text { PWS: } \mathrm{n}=27 \\
\text { Without PWS: } \\
\quad \mathrm{n}=56\end{array}$ & $\begin{array}{l}\text { PWS: } 41.5 \mathrm{~kg} / \mathrm{m}^{2}(95 \% \mathrm{CI}, 38.5-44.6) \text {; } \\
55.6 \%(95 \% \mathrm{CI}, 54.1-57.1) \\
\text { Without PWS: } 41.8 \mathrm{~kg} / \mathrm{m}^{2}(95 \% \mathrm{CI}, \\
\quad 40.9-42.8) ; 51 \%(95 \% \mathrm{CI}, 50-52.1)\end{array}$ \\
\hline 2 & $\begin{array}{l}\text { Older Icelandic adults } \\
\quad(65+\text { years })\end{array}$ & $\begin{array}{l}\text { M: } 75 \pm 6 \\
\text { F: } 72 \pm 5\end{array}$ & $\begin{array}{l}M: n=41 \\
F: n=57\end{array}$ & $\begin{array}{l}\text { M: } 30.1 \pm 4.9 \mathrm{~kg} / \mathrm{m}^{2}, 33.3 \% \pm 5.5 \% \\
\mathrm{~F}: 27.9 \pm 5.3 \mathrm{~kg} / \mathrm{m}^{2}, 40.6 \% \pm 6.3 \%\end{array}$ \\
\hline 3 & Obese European F & $\begin{array}{l}\text { Lunar: } 37(95 \% \text { CI, 35-39) } \\
\text { Hologic: } 34(95 \% \text { CI, 33-36) } \\
\text { (range for all: } 20-50)\end{array}$ & $\begin{array}{l}\mathrm{n}=47 \\
\quad(\text { Lunar }) \\
\mathrm{n}=84 \\
\quad(\text { Hologic })\end{array}$ & $\begin{array}{l}\text { Lunar: } 33.8 \mathrm{~kg} / \mathrm{m}^{2}(95 \% \mathrm{CI}, 32.9-34.8) \\
\text { Hologic: } 35.7 \mathrm{~kg} / \mathrm{m}^{2}(95 \% \mathrm{CI}, 34.9-36.6) \\
\text { Range for all: } 28.1-45 \mathrm{~kg} / \mathrm{m}^{2}\end{array}$ \\
\hline 4 & $\begin{array}{l}\text { Healthy F American } \\
\text { children and adults }\end{array}$ & $\begin{array}{l}\text { Caucasians: } 42.4 \pm 19.5 \\
\text { African Americans: } 51.7 \pm \\
\quad 18.4 \text { (range for all: } 12-94 \text { ) }\end{array}$ & $\begin{array}{l}\text { Caucasians: } \\
\mathrm{n}=785 \\
\text { African } \\
\quad \text { Americans: } \\
\mathrm{n}=159\end{array}$ & $\begin{array}{l}\text { Caucasians: } 24.5 \pm 5.3 \mathrm{~kg} / \mathrm{m}^{2} \\
\text { African Americans: } 28.1 \pm 5.7 \mathrm{~kg} / \mathrm{m}^{2}\end{array}$ \\
\hline 5 & $\begin{array}{l}\text { Healthy Swedish older } \\
\text { adults from the NORA75 } \\
\text { birth cohort study }\end{array}$ & 75 & $\begin{array}{l}M: n=51 \\
F: n=55\end{array}$ & $\begin{array}{l}\text { M: } 25.6 \pm 2.9 \mathrm{~kg} / \mathrm{m}^{2} \\
\mathrm{~F}: 25.3 \pm 3.8 \mathrm{~kg} / \mathrm{m}^{2} \\
\left(\text { range for all: } 19.2-33.6 \mathrm{~kg} / \mathrm{m}^{2} \text { ) }\right.\end{array}$ \\
\hline 6 & $\begin{array}{l}\text { Older adults from } \\
\text { Framingham Heart Study }\end{array}$ & $\begin{array}{l}\text { M: } 78 \pm 4 \\
\text { F: } 78 \pm 5\end{array}$ & $\begin{array}{l}M: n=161 \\
F: n=294\end{array}$ & $\begin{array}{l}\text { M: } 28 \pm 3.7 \mathrm{~kg} / \mathrm{m}^{2}, 30.7 \% \pm 7 \% \\
\mathrm{~F}: 27.1 \pm 4.7 \mathrm{~kg} / \mathrm{m}^{2}, 40.6 \% \pm 7.6 \%\end{array}$ \\
\hline 7 & Healthy Dutch subjects ${ }^{2 b}$ & $\begin{array}{l}28 \pm 17(\mathrm{M} / \mathrm{F} \text { breakdown not } \\
\text { provided })\end{array}$ & $\mathrm{n}=661$ & $\begin{array}{l}22.2 \pm 4.2 \mathrm{~kg} / \mathrm{m} 2 \text { (range: } 13.9-40.9) \\
24.6 \% \pm 9.1 \%\end{array}$ \\
\hline 8 & Healthy Dutch older adults & $\begin{array}{l}\text { M: } 70 \pm 5 \\
\text { F: } 68 \pm 5 \\
\text { (range for all: } 60-83 \text { ) }\end{array}$ & $\begin{array}{l}M: n=35 \\
F: n=37\end{array}$ & $\begin{array}{l}\text { M: } 25 \pm 2.2 \mathrm{~kg} / \mathrm{m}^{2}, 31 \% \pm 4.5 \% \\
\mathrm{~F}: 25.9 \pm 3.2 \mathrm{~kg} / \mathrm{m}^{2}, 43.9 \% \pm 4.3 \%\end{array}$ \\
\hline 9 & Healthy Danish adults ${ }^{\mathrm{a}}$ & $\begin{array}{l}M: 53 \pm 12(35-65) \\
F: 52 \pm 10(35-65)\end{array}$ & $\begin{array}{l}M: n=72 \\
F: n=67\end{array}$ & $\begin{array}{l}\mathrm{M}: 29.1 \mathrm{~kg} / \mathrm{m}^{2}, 27.7 \% \pm 7 \%(6.7-41.8) \\
\mathrm{F}: 28.9 \mathrm{~kg} / \mathrm{m}^{2}, 37.8 \% \pm 8.2 \%(19.1-51.3)\end{array}$ \\
\hline 10 & Healthy American adults & $\begin{array}{l}\text { M: } 35 \pm 6 \\
\text { F: } 45 \pm 3 \\
\text { (range for all: } 20-73 \text { ) }\end{array}$ & $\begin{array}{l}M: n=51 \\
F: n=59\end{array}$ & $\begin{array}{l}\text { M: } 26.2 \mathrm{~kg} / \mathrm{m}^{2}, 25 \% \\
\text { F: } 25 \mathrm{~kg} / \mathrm{m}^{2}, 37 \%\end{array}$ \\
\hline 11 & Healthy American F & $\mathrm{F}: 27 \pm 8$ & $\mathrm{~F}: \mathrm{n}=510$ & $23.6 \mathrm{~kg} / \mathrm{m}^{\mathrm{b}}, 27.8 \% \pm 8 \%$ \\
\hline 12 & Healthy American adults & $\begin{array}{l}\text { M: } 33 \pm 10 \\
F: 35 \pm 9 \text { (range for all: } 18-64)\end{array}$ & $\begin{array}{l}M: n=123 \\
F: n=65\end{array}$ & $\begin{array}{l}\text { M: } 23.9 \mathrm{~kg} / \mathrm{m}^{2} \\
\mathrm{~F}: 21.9 \mathrm{~kg} / \mathrm{m}^{2}\end{array}$ \\
\hline 13 & Healthy American adults & $\begin{array}{l}\text { M: } 27 \pm 8 \text { (range: } 18-50) \\
F: 27 \pm 6 \text { (range: } 19-43)\end{array}$ & $\begin{array}{l}M: n=47 \\
F: n=67\end{array}$ & $\begin{array}{l}\text { M: } 25.8 \mathrm{~kg} / \mathrm{m}^{2}, 16.2 \% \pm 7 \% \\
F: 22.3 \mathrm{~kg} / \mathrm{m}^{2}, 25.1 \% \pm 6.6 \%\end{array}$ \\
\hline
\end{tabular}

CI, confidence interval; BMI, body mass index; F, female; M, male; PWS, Prader-Willi Syndrome.

${ }^{\mathrm{a}}$ Measured using reference technique.

${ }^{\mathrm{b}}$ Equation was developed using only subjects aged 16-83 years; however, age, BMI, and percent body fat data in table include subjects aged 7-15 years.

\section{Bland-Altman Analysis}

Proportional bias was not evident in equations 1, 4, 5, 6, 7, 8, 9a, 9b, 10, and 11b (Supplementary Figure S3). Fixed bias (ie, the mean difference between the 2 methods) was within $0.0 \pm 0.504 \mathrm{~kg}$ only for equation 10 (Figure 3). No equations had limits of agreement within $\pm 10 \%$.

\section{Ranking of Equations}

Equation 10 met 3 of the 6 criteria, and equations 6 and 7 met 2 of the 6 criteria. Equations 1, 4, 5, 8, 9a, 9b, and $11 \mathrm{~b}$ met 1 criterion, and the remaining equations met none (Table 6).

\section{Comparison of FFM Across BMI Categories}

FFM $_{\mathrm{DXA}}$ and $\mathrm{FFM}_{\mathrm{BI}}$ increased along with BMI category, such that both were greater in patients with overweight vs normal weight and greater in those with obesity vs overweight (Table 7). However, FFM DXA $_{\text {and FFM }}$ BIA were statistically similar between patients with obesity and severe obesity. The mean difference between the 2 methods $\left(F_{F M} M_{\text {BIA }}-\right.$ FFM $\left._{\mathrm{DXA}}\right)$ was significantly smaller in patients with severe obesity $(-0.2 \pm 3.1 \mathrm{~kg})$ compared with all other BMI categories. The mean differences between the 2 methods $\left(\mathrm{FFM}_{\mathrm{BIA}}-\mathrm{FFM}_{\mathrm{DXA}}\right)$ were not statistically different between patients with normal weight, overweight, and obesity. 
Table 5. Comparison Summary of Equation-Generated Estimates of FFM BIA $_{\text {. }}$

\begin{tabular}{|c|c|c|c|c|c|c|}
\hline \multirow[b]{2}{*}{ Equation } & \multirow{2}{*}{$\begin{array}{c}\text { Mean } \pm \text { SD } \\
\mathrm{kg}\end{array}$} & \multirow{2}{*}{$\begin{array}{l}\text { Significance } \\
\text { of Difference } \\
(P \text {-Value })^{\mathrm{a}}\end{array}$} & \multirow{2}{*}{$\begin{array}{c}\text { Concordance } \\
\text { Correlation } \\
\left(r_{\mathrm{c}}\right)\end{array}$} & \multirow{2}{*}{$\begin{array}{l}\text { Fixed Bias, } \\
\text { kg }\end{array}$} & \multicolumn{2}{|c|}{ Limits of Agreement } \\
\hline & & & & & $\mathrm{kg}$ & $\pm \%$ \\
\hline $\begin{array}{l}\text { Reference method } \\
\text { (DXA) }\end{array}$ & $42 \pm 5.9$ & - & - & - & - & - \\
\hline 1 & $52.2 \pm 5.6$ & $<.0001$ & 0.11 & 10.2 & -0.6 to 21 & 26 \\
\hline 2 & $43.2 \pm 4.4$ & .002 & 0.56 & 1.3 & -3.9 to 6.4 & 12 \\
\hline $3 \mathrm{a}$ & $48.8 \pm 4.7$ & $<.0001$ & 0.23 & 6.9 & -0.1 to 13.9 & 17 \\
\hline $3 b$ & $51.9 \pm 8$ & $<.0001$ & 0.13 & 9.9 & -3.6 to 23.4 & 32 \\
\hline 4 & $47.9 \pm 6.9$ & $<.0001$ & 0.30 & 6.0 & -3 to 14.9 & 21 \\
\hline 5 & $48.1 \pm 6$ & $<.0001$ & 0.27 & 6.1 & -2.4 to 14.6 & 20 \\
\hline 6 & $43.0 \pm 5.3$ & .071 & 0.50 & 1.1 & -6.9 to 9.1 & 19 \\
\hline 7 & $43.8 \pm 6.6$ & .001 & 0.82 & 1.8 & -4.8 to 8.5 & 16 \\
\hline 8 & $39.6 \pm 6.7$ & .009 & 0.36 & -2.4 & -14.2 to 9.5 & 28 \\
\hline $9 \mathrm{a}$ & $47.2 \pm 6.1$ & $<.0001$ & 0.37 & 5.2 & -1 to 11.5 & 15 \\
\hline $9 b$ & $46.6 \pm 5.2$ & $<.0001$ & 0.36 & 4.7 & -1.8 to 11.1 & 15 \\
\hline 10 & $41.9 \pm 6.9$ & .937 & 0.56 & -0.1 & -9 to 8.9 & 21 \\
\hline $11 \mathrm{a}$ & $45.8 \pm 5.2$ & $<.0001$ & 0.43 & 3.9 & -1.5 to 9.2 & 13 \\
\hline $11 \mathrm{~b}$ & $44.6 \pm 5.4$ & $<.0001$ & 0.54 & 2.4 & -2.2 to 7 & 11 \\
\hline $12 \mathrm{a}$ & $44.7 \pm 7.2$ & $<.0001$ & 0.58 & 2.7 & -3.5 to 8.9 & 15 \\
\hline $12 b$ & $43.0 \pm 7$ & .028 & 0.66 & 1 & -5 to 7 & 14 \\
\hline $13 \mathrm{a}$ & $48.6 \pm 8.2$ & $<.0001$ & 0.21 & 6.6 & -7.1 to 20.3 & 33 \\
\hline $13 b$ & $48.6 \pm 8$ & $<.0001$ & 0.26 & 6.7 & -5 to 18.3 & 28 \\
\hline
\end{tabular}

BIA, bioelectrical impedance analysis; DXA, dual-energy X-ray absorptiometry; FFM, fat-free mass.

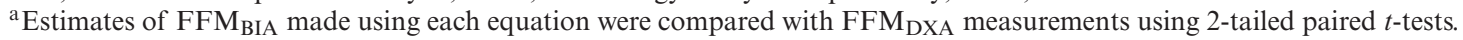

In the normal weight, overweight, and obese subgroups of patients, 16 and 17 equations overestimated FFM $_{\mathrm{BIA}}$, and $1-2$ equations underestimated $\mathrm{FFM}_{\mathrm{BIA}}$, relative to DXA. In the group of patients with severe obesity, 10 equations overestimated $\mathrm{FFM}_{\mathrm{BIA}}$, and 8 equations underestimated FFM $_{\text {BIA }}$. Notably, the equations that performed the best ( 3 points) and worst ( 0 points) were not consistent across BMI categories.

\section{Discussion}

Despite the widespread use of BIA in clinical populations, we are the first to comprehensively evaluate the accuracy of several BIA prediction equations against a reference method (DXA) in breast cancer patients currently undergoing treatment. In the present study, we found that most equations overestimated FFM by an average of $\approx 4.1 \mathrm{~kg}$ in this group of breast cancer patients. Our results suggest that researchers and clinicians who are forced to rely exclusively on BIA to assess body composition in breast cancer patients should use equations $6,{ }^{23} 7,{ }^{24}$ or $10^{25}$; however, even these top-ranked equations did not produce estimations that aligned very closely with DXA. All other equations are not recommended for this population, but especially $2,{ }^{26} 3 \mathrm{a},{ }^{27}$ $3 b,{ }^{27} 11 a,{ }^{28} 12 a,{ }^{29} 12 b,{ }^{29} 13 a,{ }^{30}$ and $13 b,{ }^{30}$ because they met none of our accuracy criteria.

The majority of BIA prediction equations produced overestimates of FFM ranging from 1 to $10.2 \mathrm{~kg}$, relative to
DXA. The tendency to overestimate FFM directly results in an underestimation of FM, suggesting that BIA assessments of adiposity measured in clinic may be inaccurate in this patient population. This is concerning, because studies employing BIA regularly report whole-body FM as high as $40 \%$ in breast cancer patients during treatment ${ }^{31,32}$ and in survivorship. ${ }^{33,34}$ Given the underestimation of adiposity predicted by our study, these patients may therefore be at greater risk of developing secondary comorbidities related to excess adiposity, such as cardiovascular disease and diabetes, than previously thought. Further, consistent underestimation of FM in clinic and research may indicate a need for reevaluation of the effectiveness of existing weight-management recommendations and breast cancer rehabilitation programs.

We identified 3 BIA prediction equations that agreed most closely with DXA measurements of FFM, yet even these comparatively high-performing equations did not fulfill all of the predetermined accuracy criteria, making it difficult to advocate for their use in this clinical population. In particular, the limits of agreement during Bland-Altman analysis for these 3 equations were fairly wide and ranged from $\pm 15 \%$ to $19 \%$. Schubert et $\mathrm{al}^{35}$ have shown that the limits of agreement for repeat DXA scans and for DXA vs MF-BIA are $\approx \pm 6 \%$ and $\pm 7 \%$, respectively, in young healthy adults. Although there is no consensus on clinically acceptable limits of agreement, values $> \pm 5 \%-10 \%$ have 


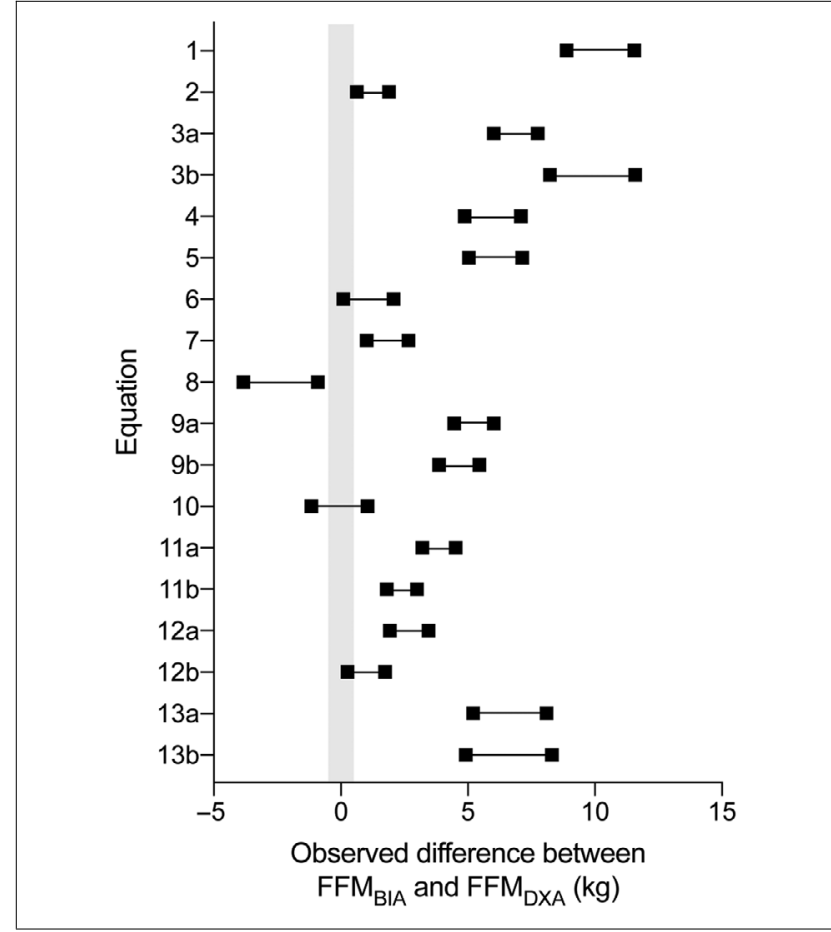

Figure 1. Equivalence testing of the prediction equations. Equations were considered equivalent to the reference method (ie, DXA) if the $90 \%$ CI (indicated by the black symbols and lines) of the mean difference between FFM $_{\text {BIA }}$ and FFM ${ }_{\text {DXA }}$ was completely contained within the equivalence region of -0.504 to $0.504 \mathrm{~kg}$ (indicated by the gray-shaded area). BIA, bioelectrical impedance analysis; CI, confidence interval; DXA, dual-energy x-ray absorptiometry.

previously been cited as "wide." 12,20 Considering that the limits of agreement for the 3 equations that performed best in the present study fall outside this range, it is difficult to advocate for their use in clinic. All 3 equations were derived using the RJL BIA-101 but were validated against different reference techniques $\left(\mathrm{DXA}^{23}\right.$ [equation 6], hydrodensitometry ${ }^{24}$ [equation 7], and deuterium dilution ${ }^{25}$ [equation 10]). The populations used to develop the equations varied: Roubenoff et $\mathrm{al}^{23}$ (equation 6) recruited older adults with overweight, whereas Deurenberg et $\mathrm{al}^{24}$ (equation 7) and Lukaski et $\mathrm{al}^{25}$ (equation 10) both included healthy adults. The equations that performed the worst in our group of patients were not united by common characteristics. It is perhaps unsurprising that we were unable to identify a single equation that accurately predicted FFM in this cohort, because breast cancer patients often violate 2 common assumptions of whole-body tetrapolar SF-BIA measurements. First, SF-BIA considers the limbs and the trunk to be concentric cylinders of subcutaneous adipose tissue, muscle, and bone that function as a circuit of parallel resistors to an electric current. ${ }^{36}$ However, this assumption is potentially problematic in individuals with obesity, and previous work has shown that excess adiposity (particularly abdominal adiposity) results in overestimations of FFM relative to reference methods such as hydrostatic weighing ${ }^{37}$ and magnetic resonance imaging. ${ }^{38}$ Furthermore, BIA prediction equations that were derived and validated in normalweight individuals tend to overestimate FFM in healthy individuals with obesity. ${ }^{36}$ Given that our patients had, on average, overweight and central obesity, they likely had increased FM over the trunk and limbs, resulting in overestimates of FFM with the previously published prediction equations. Secondly, BIA assumes normal hydration of lean tissues, as well as an even distribution of fluid between the extracellular and intracellular compartments. Although breast cancer patients sometimes develop lymphedema following surgery (specifically, the removal of axillary lymph nodes), only 2 of the patients in this study exhibited overt signs of lymphedema. Given that lymphedema typically

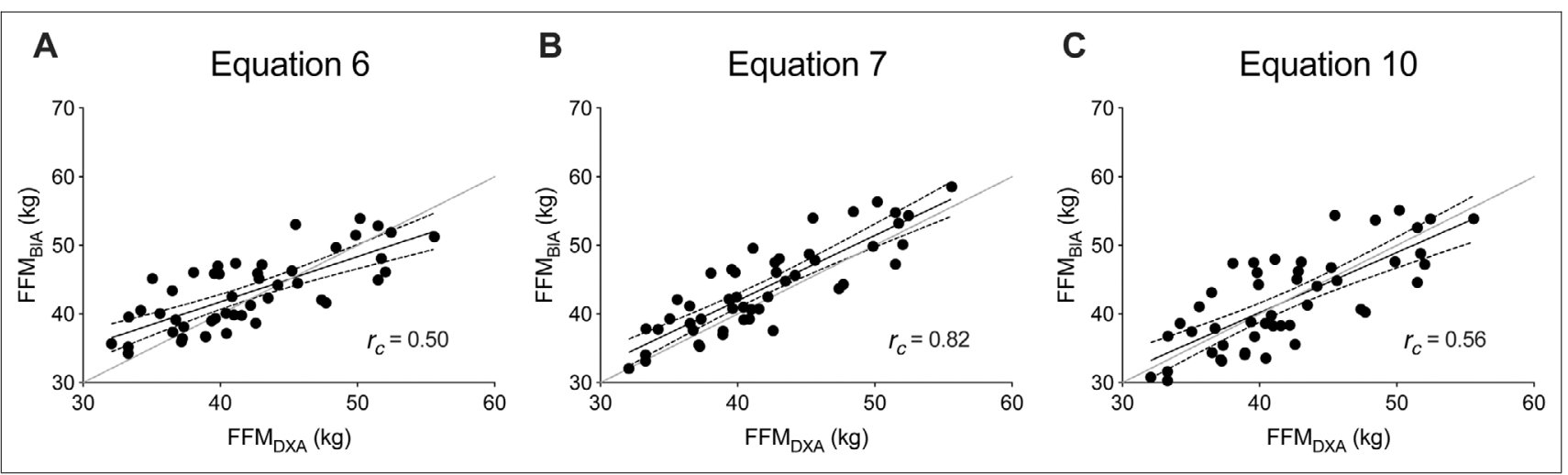

Figure 2. Correlation analysis for the top-ranked equations: (A) equation 6; (B) equation 7; and (C) equation 10. We conducted Lin's concordance correlations $\left(r_{\mathrm{c}}\right)$ between $\mathrm{FFM}_{\mathrm{DXA}}$ and estimates of $\mathrm{FFM}_{\mathrm{BIA}}$ generated using these equations. On each panel, the solid black line and dashed curved lines represent the line of best fit and $95 \%$ confidence intervals, respectively. The solid gray line is the $45^{\circ}$ line of identity $(y=x)$, which represents perfect agreement between 2 variables. BIA, bioelectrical impedance analysis; DXA, dual-energy x-ray absorptiometry; FFM, fat-free mass. 
Table 6. Summary of Statistical Analysis.

\begin{tabular}{|c|c|c|c|c|c|c|c|}
\hline \multirow[b]{2}{*}{ Equation } & \multirow{2}{*}{$\begin{array}{c}\frac{\text { Paired } t \text {-Test }}{} \\
P>.05\end{array}$} & \multirow{2}{*}{$\begin{array}{c}\text { Correlation } \\
r_{\mathrm{c}}>0.80\end{array}$} & \multirow{2}{*}{$\begin{array}{c}\text { Equivalence Test } \\
\text { 90\% CI Contained } \\
\text { Within }-0.504 \text { to } \\
0.504 \mathrm{~kg}\end{array}$} & \multicolumn{3}{|c|}{ Bland-Altman Analysis } & \multirow[b]{2}{*}{$\begin{array}{l}\text { Points } \\
\text { Total }\end{array}$} \\
\hline & & & & $\begin{array}{l}\text { Small Fixed Bias } \\
(0 \pm 0.504 \mathrm{~kg})\end{array}$ & $\begin{array}{c}\text { Narrow LOA } \\
\text { (Within } \pm 10 \% \text { ) }\end{array}$ & $\begin{array}{c}\text { No Proportional } \\
\text { Bias }\end{array}$ & \\
\hline 1 & & & & & & $\boldsymbol{V}$ & 1 \\
\hline 2 & & & & $\mathrm{n} / \mathrm{a}$ & & & 0 \\
\hline $3 a$ & & & & $\mathrm{n} / \mathrm{a}$ & & & 0 \\
\hline $3 b$ & & & & $\mathrm{n} / \mathrm{a}$ & & & 0 \\
\hline 4 & & & & & & $\checkmark$ & 1 \\
\hline 5 & & & & & & $\checkmark$ & 1 \\
\hline 6 & $\checkmark$ & & & & & $\checkmark$ & 2 \\
\hline 7 & & $\checkmark$ & & & & $\checkmark$ & 2 \\
\hline 8 & & & & & & $\boldsymbol{V}$ & 1 \\
\hline $9 \mathrm{a}$ & & & & & & $\boldsymbol{V}$ & 1 \\
\hline $9 b$ & & & & & & $\checkmark$ & 1 \\
\hline 10 & $\checkmark$ & & & $\checkmark$ & & $\boldsymbol{V}$ & 3 \\
\hline $11 \mathrm{a}$ & & & & $\mathrm{n} / \mathrm{a}$ & & & 0 \\
\hline $11 b$ & & & & & & $\boldsymbol{V}$ & 1 \\
\hline $12 \mathrm{a}$ & & & & $\mathrm{n} / \mathrm{a}$ & & & 0 \\
\hline $12 b$ & & & & $\mathrm{n} / \mathrm{a}$ & & & 0 \\
\hline $13 \mathrm{a}$ & & & & $\mathrm{n} / \mathrm{a}$ & & & 0 \\
\hline $13 b$ & & & & $\mathrm{n} / \mathrm{a}$ & & & 0 \\
\hline
\end{tabular}

Equations were assigned a point for each of the 6 criteria they met.

CI, confidence interval; LOA, limits of agreement.

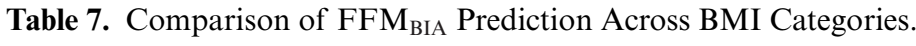

\begin{tabular}{|c|c|c|c|c|c|}
\hline Measurement & All Patients & $\begin{array}{c}\text { Normal } \\
\left(18.5-24.9 \mathrm{~kg} / \mathrm{m}^{2}\right)\end{array}$ & $\begin{array}{c}\text { Overweight } \\
\left(25-29.9 \mathrm{~kg} / \mathrm{m}^{2}\right)\end{array}$ & $\begin{array}{l}\text { Obese }(30-35 \\
\left.\mathrm{kg} / \mathrm{m}^{2}\right)\end{array}$ & $\begin{array}{c}\text { Severely Obese } \\
\left(>35 \mathrm{~kg} / \mathrm{m}^{2}\right)\end{array}$ \\
\hline $\mathrm{FFM}_{\mathrm{DXA}}, \mathrm{kg}^{\mathrm{a}}$ & $\begin{array}{c}42 \pm 5.9 \\
(32.1-55.6)\end{array}$ & $\begin{array}{c}38 \pm 3.6^{\mathrm{a}} \\
(33.3-45.5)\end{array}$ & $\begin{array}{l}41.8 \pm 5.1^{b} \\
(32.1-51.5)\end{array}$ & $\begin{array}{l}46.3 \pm 5.5^{\mathrm{c}} \\
(39.4-55.6)\end{array}$ & $\begin{array}{l}50.6 \pm 2.8^{c} \\
(47.4-52.5)\end{array}$ \\
\hline $\mathrm{FFM}_{\mathrm{BIA}}, \mathrm{kg}^{\mathrm{a}}$ & $\begin{array}{l}46.1 \pm 3.4 \\
(39.6-52.2)\end{array}$ & $\begin{array}{c}43.2 \pm 4^{\mathrm{a}} \\
(37.9-51.2)\end{array}$ & $\begin{array}{l}45.8 \pm 3.4^{b} \\
(39.1-51.8)\end{array}$ & $\begin{array}{c}50 \pm 3.1^{\mathrm{c}} \\
(41.7-54.4)\end{array}$ & $\begin{array}{l}50.4 \pm 3.1^{\mathrm{c}} \\
(41.4-54.1)\end{array}$ \\
\hline $\mathrm{FFM}_{\mathrm{BIA}}-\mathrm{FFM}_{\mathrm{DXA}}, \mathrm{kg}^{\mathrm{a}}$ & $\begin{array}{c}4.1 \pm 3.4 \\
(-2.4 \text { to } 10.2)\end{array}$ & $\begin{array}{c}5.2 \pm 4^{\mathrm{a}} \\
(-0.1 \text { to } 13.2)\end{array}$ & $\begin{array}{c}4 \pm 3.4^{\mathrm{a}} \\
(-2.7 \text { to } 10)\end{array}$ & $\begin{array}{l}3.7 \pm 3.1^{\mathrm{a}} \\
(-4.6 \text { to } 8)\end{array}$ & $\begin{array}{l}-0.2 \pm 3.1^{\mathrm{b}} \\
(-9.2 \text { to } 3.4)\end{array}$ \\
\hline \multicolumn{6}{|l|}{ Prediction equations } \\
\hline \# Overestimating FFM & 16 & 17 & 16 & 16 & 10 \\
\hline \# Underestimating FFM & 2 & 1 & 2 & 2 & 8 \\
\hline With 0 points & $\begin{array}{l}2,3 a, 3 b, 11 a, 12 a, \\
12 b, 13 a, 13 b\end{array}$ & $1,2,3 b, 4,13 a, 13 b$ & $3 b, 13 a, 13 b$ & 2 & - \\
\hline With 3 points & 10 & $12 b$ & 10 & $\begin{array}{c}6,9 \mathrm{~b}, 10,11 \mathrm{a}, 11 \mathrm{~b} \\
12 \mathrm{a}, 12 \mathrm{~b}\end{array}$ & $\begin{array}{c}3 \mathrm{a}, 9 \mathrm{a}, 9 \mathrm{~b}, 11 \mathrm{a}, \\
13 \mathrm{~b}\end{array}$ \\
\hline
\end{tabular}

Data are mean \pm SD (range), unless otherwise indicated.BIA, bioelectrical impedance analysis; BMI, body mass index; DXA, dual-energy x-ray absorptiometry; FFM, fat-free mass. Values that do not share a letter $(\mathrm{a}, \mathrm{b}, \mathrm{c}$, or d) are statistically different $(\mathrm{P}<0.001)$.

a $(P<.001)$.

occurs in $\approx 15 \%$ of breast cancer patients, ${ }^{39}$ there were likely a number of patients in the current study with subclinical lymphedema. However, hydration status and fluid distribution may become altered even in patients without lymphedema. Recent work has shown that systemic therapies, such as certain types of chemotherapy and hormonal treatment, may independently lead to mild swelling of the trunk and upper extremities as the result of water retention, ${ }^{40-42}$ likely altering mass distribution. In order to confidently and accurately assess FFM (and FM) in breast cancer patients and survivors using BIA, new equations developed in these distinct patient populations are required.

A key comparison can be made between the present study and that of Lee et $a 1,{ }^{33}$ who also evaluated BIA estimates of FFM against DXA-derived measurements in a group of similarly aged (53 \pm 10 [range: 29-76] years) 


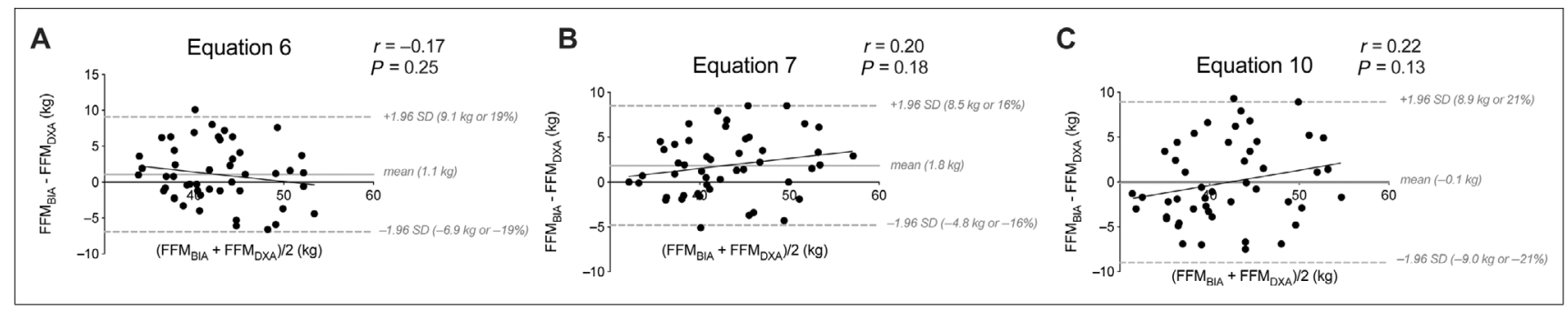

Figure 3. Bland-Altman plots for the top-ranked equations: (A) equation 6; (B) equation 7; and (C) equation 10. Note the

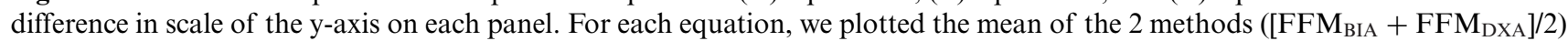
against the difference between the 2 methods $\left(\mathrm{FFM}_{\mathrm{BIA}}-\mathrm{FFM}_{\mathrm{DXA}}\right)$. To test for proportional bias, we conducted 2-tailed Pearson correlations between the mean of and difference between the 2 methods. Correlation coefficients $(r)$ and $P$-values for this relationship are presented on each graph. The solid black line is the line of best fit. The solid gray line indicates fixed bias (ie, the mean difference), and the dashed gray lines indicate the LOA (ie, mean difference $\pm 1.96 \mathrm{SD}$ ) in absolute values and as a

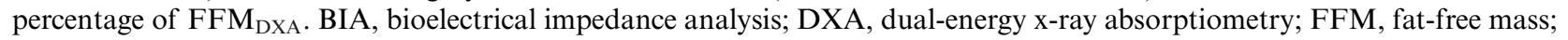
LOA, limits of agreement.

women who had recently completed treatment for breast cancer. Consistent with our findings, Lee et $\mathrm{al}^{33}$ observed that BIA overestimated FFM relative to DXA across all patients $(+3.8 \mathrm{~kg})$ and also that BIA performed significantly better in patients with severe obesity $\left(>35 \mathrm{~kg} / \mathrm{m}^{2}\right)$ compared with patients with lower BMIs $\left(\leq 35 \mathrm{~kg} / \mathrm{m}^{2}\right)$. The patients with severe obesity had more FFM (as measured by DXA) than all other patients, which is likely because of compensatory hypertrophy of skeletal muscle in response to the demands of supporting excess body mass. ${ }^{43}$ Importantly, elevated FFM in these patients may have contributed to the improved agreement between BIA and DXA in this patient subgroup. Indeed, in the current study, we observed that FFM increased in a stepwise fashion as BMI category progressed from normal to severe obesity, possibly reducing the degree to which BIA overestimated FFM in patients with excess adiposity. However, because patients with severe obesity comprised a minority of the overall patient sample in both our study and Lee et al, ${ }^{33}$ it is possible that the superior agreement between BIA and DXA may have been the result of a small and skewed data set. Future studies with larger sample sizes are needed to verify this potential effect.

Despite these similarities, several important distinctions must be drawn between the current study and Lee et al. ${ }^{33}$ Lee et $\mathrm{al}^{33}$ measured percent body fat in breast cancer survivors on an MF-BIA (InBody 520 Body Composition Analyzer; BioSpace, Cerritos, CA, USA) using the manufacturer's proprietary equation and subsequently used body mass to indirectly calculate FM and FFM. In the current study, we examined a distinct clinical cohort (patients currently undergoing treatment for breast cancer) using an alternative, more rigorous approach. We applied raw $R$ and $X$ data generated using SF-BIA (which is more commonly used in clinic, compared with MF-BIA) to an array of previously published prediction equations, which disclose the characteristics of the populations in which they were derived and validated. Further, based on recent recommendations aimed at improving comparisons between bedside (BIA) and reference (DXA) body composition technologies, ${ }^{12}$ we employed multiple statistical techniques in addition to Bland-Altman analysis. As such, although our findings complement those of Lee et al, ${ }^{33}$ our more comprehensive analysis strengthens the argument against using BIA to measure FFM in breast cancer patients. ${ }^{44}$

The findings reported herein have important implications for researchers and clinicians, but readers should be aware of several limitations of the present study. Previously published BIA prediction equations are highly specific to the population and reference modality with which they were generated. ${ }^{13}$ Though we only included equations derived in predominantly Caucasian adult populations from developed nations, many equations employed reference techniques other than the Hologic Discovery QDR 4500 DXA, which likely introduced scaling errors into our comparisons. Furthermore, although DXA generally produces moreprecise FFM measurements than BIA and is considered a reference tool, it is not error-free. In the current study, we performed all BIA measurements on the right side of the body, regardless of limb dominance, which side was affected by cancer, or whether the patient displayed overt signs of lymphedema. As such, we are unable to determine whether any of these potential confounding factors influenced the prediction of FFM by BIA. Our sample size was relatively small, but the characteristics of the patients included in the present study are similar to previously published studies on larger cohorts of breast cancer patients (ie, mean age and percent body fat, distribution across BMI categories, etc). ${ }^{2}$ Therefore, our sample of patients is reasonably representative of this clinical population, which improves the generalizability of our findings; however, larger-scale studies in the future would be clearer and more definitive. A final potential limitation of this study is that because of our small sample size, we elected to evaluate the accuracy 
of previously published BIA prediction equations rather than developing and validating a breast cancer prediction equation of our own.

In conclusion, BIA consistently overestimated FFM (resulting in underestimates of FM) in breast cancer patients currently undergoing treatment. Notably, these trends were inconsistent across BMI categories and equations. Although we highlight certain equations that may be preferable (eg, equations $6,{ }^{23} 7,,^{24}$ or $10^{25}$ ) compared with others, caution should be used when interpreting body composition outcomes measured using BIA in breast cancer patients. Considering the positive associations between excess adiposity and breast cancer incidence, as well as the role that deleterious body composition changes play in the development of comorbid conditions and breast cancer recurrence, there is an urgent need for clinically accessible and accurate technologies. Future studies are required to develop and validate BIA prediction equations specific to breast cancer patients throughout the disease trajectory.

\section{Statement of Authorship}

S. Schmidt and M. Mourtzakis contributed to the conception and design of the research; K. E. Bell, S. Schmidt, A. Pfeiffer, L. Bos, and C. Russell contributed to the acquisition and analysis of the data; K. E. Bell, C. Earthman, and M. Mourtzakis contributed to the interpretation of the data; and K. E. Bell drafted the manuscript. All authors critically revised the manuscript, agree to be fully accountable for ensuring the integrity and accuracy of the work, and read and approved the final manuscript.

\section{Supplementary Information}

Additional supporting information may be found online in the Supporting Information section at the end of the article.

\section{References}

1. Amaral P, Miguel R, Mehdad A, et al. Body fat and poor diet in breast cancer women. Nutr Hosp. 2010;25(3):456-461.

2. Caan BJ, Cespedes Feliciano EM, Prado CM, et al. Association of muscle and adiposity measured by computed tomography with survival in patients with nonmetastatic breast cancer. JAMA Oncol. 2018;4(6):798-804.

3. Yaw Y, Kandiah M, Shariff Z, et al. Pattern of weight changes in women with breast cancer. Asian Pac J Cancer Prev. 2010;11(6): $1535-1540$

4. Wahnefried W, Rimer BK, Winer EP. Weight gain in women diagnosed with breast cancer. J Am Diet Assoc. 1997;97(5):519-529.

5. Vance V, Mourtzakis M, McCargar L, Hanning R. Weight gain in breast cancer survivors: prevalence, pattern and health consequences. Obes Rev. 2011;12(4):282-294.

6. Chan DS, Vieira AR, Aune D, et al. Body mass index and survival in women with breast cancer-systematic literature review and metaanalysis of 82 follow-up studies. Ann Oncol. 2014;25(10):1901-1914.

7. Litton JK, Gonzalez-Angulo AM, Warneke CL, et al. Relationship between obesity and pathologic response to neoadjuvant chemotherapy among women with operable breast cancer. J Clin Oncol. 2008;26(25):4072-4077.
8. Ioannides SJ, Barlow PL, Elwood JM, Porter D. Effect of obesity on aromatase inhibitor efficacy in postmenopausal, hormone receptorpositive breast cancer: a systematic review. Breast Cancer Res Treat. 2014;147(2):237-248.

9. Demark-Wahnefried W, Campbell KL, Hayes SC. Weight management and its role in breast cancer rehabilitation. Cancer. 2012;118(8 suppl):2277-2287.

10. Kutynec CL, McCargar L, Barr SI, Hislop TG. Energy balance in women with breast during adjuvant treatment. $J$ Am Diet Assoc. 1999;99(10):1222-1227.

11. Thomson ZO, Reeves MM. Can weight gain be prevented in women receivingtreatment for breast cancer? a systematic review ofintervention studies. Obes Rev. 2017;18(11):1364-1373.

12. Earthman C. Body composition tools for assessment of adult malnutrition at the bedside: a tutorial on research considerations and clinical applications. J Parenter Enteral Nutr. 2015;39(7):787-822.

13. Graves JE, Pollock ML, Colvin AB, Van Loan M, Lohman TG. Comparison of different bioelectrical impedance analyzers in the prediction of body composition. Am J Hum Biol. 1989;1(5) 603-611.

14. Nana A, Slater GJ, Hopkins WG, Burke LM. Techniques for undertaking dual-energy $\mathrm{X}$-ray absorptiometry whole-body scans to estimate body composition in tall and/or broad subjects. Int J Sport Nutr Exerc Metab. 2012;22(5):313-322.

15. Dixon PM, Saint-Maurice PF, Kim Y, Hibbing P, Bai Y, Welk GJ. A Primer on the use of equivalence testing for evaluating measurement agreement. Med Sci Sports Exerc. 2018;50(4):837-845.

16. Carver TE, Christou NV, Andersen RE. In vivo precision of the GE iDXA for the assessment of total body composition and fat distribution in severely obese patients. Obesity. 2013;21(7):1367-1369.

17. Cordero-MacIntyre ZR, Peters W, Libanati CR, et al. Reproducibility of DXA in obese women. $J$ Clin Densitom. 2002;5(1):35-44.

18. Knapp KM, Welsman JR, Hopkins SJ, Shallcross A, Fogelman I, Blake GM. Obesity increases precision errors in total body dual-energy x-ray absorptiometry measurements. $J$ Clin Densitom. 2015;18(2): 209-216.

19. Bland J, Altman D. Statistical methods for assessing agreement between two methods of clinical assessment. Lancet. 1986;1(8476): 307-310.

20. Ward LC. Bioelectrical impedance analysis for body composition assessment: reflections on accuracy, clinical utility, and standardisation. Eur J Clin Nutr. 2019;73(2):194-199.

21. Sun SS, Chumlea WC, Heymsfield SB, et al. Development of bioelectrical impedance analysis prediciton equations for body composition with the use of a multicomponent model for use in epidemiologic surveys. Am J Clin Nutr. 2003;77(2):331-340.

22. Altman D. Practical Statistics for Medical Research. London: Chapman and Hall; 1991.

23. Roubenoff R, Baumgartner RN, Harris TB, et al. Application of bioelectrical impedance analysis to elderly populations. $J$ Gerontol A Biol Sci Med Sci. 1997;52(3):M129-136.

24. Deurenberg P, van der Kooy K, Leene R, Weststrate JA, Seidell JC. Sex and age-specific prediction formulas for estimating body composition from bioelectrical impedance: a cross-validation study. Int $J$ Obes 1991;15(1):17-25.

25. Lukaski HC, Bolonchuk WW. Estimation of body fluid volumes using tetrapolar bioelectrical impedance measurements. Aviat Space Environ Med. 1988;59(12):1163-1169.

26. Ramel A, Geirsdottir OG, Arnarson A, Thorsdottir I. Regional and total body bioelectrical impedance analysis compared with DXA in Icelandic elderly. Eur J Clin Nutr. 2011;65(8):978-983.

27. Verdich C, Barbe P, Petersen M, et al. Changes in body composition during weight loss in obese subjects in the NUGENOB study: comparison of bioelectrical impedance vs. dual-energy X-ray absorptiometry. Diabetes Metab. 2011;37(3):222-229. 
28. Segal KR, Van Loan M, Fitzgerald PI, Hodgdon JA, Van Italie TB. Lean body mass estimation by bioelectrical impedance analysis: a foursite cross-validation study. Am J Clin Nutr. 1988;47(1):7-14.

29. Van Loan M, Mayclin P. Bioelectrical impedance analysis: is it a reliable estimator of lean body mass and total body water. Hum Biol. 1987;59(2):299-309.

30. Lukaski HC, Bolonchuk WW, Hall CB, Siders WA. Validation of tetrapolar bioelectrical impedance method to assess human body composition. J Appl Physiol. 1986;60(4):1327-1332.

31. Anusca Martins K, Freitas-Junior R, Tronco Monego E, Rsende Paulnelli R. Anthropometry and lipid profile in women with breast cancer: a case-control study. Rev Col Bras Cir. 2012;39(5): 358-363.

32. Czerniak U, Demuth A, Skrzypczak M. Associations of physical activity and inactivity with body tissue composition among healthy Polish women and women after mastectomy. Homo. 2014;65(5): 423-431.

33. Lee K, Sami N, Sweeney FC, Dieli-Conwright CM. Body composition with dual-energy $\mathrm{x}$-ray absorptiometry and bioelectrical impedance analysis in breast cancer survivors. Nutr Clin Pract. 2019;34(3): 421-427.

34. Battaglini C, Naumann F, Groff D, Shields E, Hackney AC, Peppercorn J. Comparison of body composition assessment methods in breast cancer survivors. Oncol Nurs Forum. 2011;38(4):E283-E290.

35. Schubert MM, Seay RF, Spain KK, Clarke HE, Taylor JK. Reliability and validity of various laboratory methods of body composition assessment in young adults. Clin Physiol Funct Imaging. 2019;39(2): 150-159.

36. Deurenberg P. Limitations of the bioelectrical impedance method for the assessment of body fat in severe obesity. Am J Clin Nutr. 1996;64(3 suppl):449S-452S.
37. Gray DS, Bray GA, Kaplan K. Effect of obesity on bioelectrical impedance. Am J Clin Nutr. 1989;50(2):255-260.

38. Baumgartner RN, Ross R, Heymsfield SB. Does adipose tissue influence bioelectric impedance in obese men and women? J Appl Physiol. 1998;84(1):257-262.

39. DiSipio T, Rye S, Newman B, Hayes S. Incidence of unilateral arm lymphoedema after breast cancer: a systematic review and metaanalysis. The Lancet Oncology. 2013;14(6):500-515.

40. Zhu W, Li D, Li X, et al. Association between adjuvant docetaxelbased chemotherapy and breast cancer-related lymphedema. Anticancer Drugs. 2017;28(3):350-355.

41. Swaroop MN, Ferguson CM, Horick NK, et al. Impact of adjuvant taxane-based chemotherapy on development of breast cancer-related lymphedema: results from a large prospective cohort. Breast Cancer Res Treat. 2015;151(2):393-403.

42. Invernizzi M, Michelotti A, Noale M, et al. Breast cancer systemic treatments and upper limb lymphedema: a risk-assessment platform encompassing tumor-specific pathological features reveals the potential role of trastuzumab. J Clin Med. 2019;8(2):138.

43. Forbes GB, Welle SL. Lean body mass in obesity. Int $J$ Obes. 1983;7(2):99-107.

44. Kyle UG, Bosaeus I, De Lorenzo AD, et al. Bioelectrical impedance analysis-part II: utilization in clinical practice. Clin Nutr. 2004;23(6):1430-1453.

45. Withers RT, Laforgia J, Heymsfield SB. Critical appraisal of the estimation of body composition via two-, three-, and four-compartment models. Am J Hum Biol. 1999;11(2):175-185.

46. Bruce A, Andersson M, Arvidsson B, Isaksson B. Body composition. Prediction of normal body potassium, body water and body fat in adults on the basis of body height, body weight and age. Scand J Clin Lab Invest. 1980;40(5):461-473. 